\title{
The sum of the same powers of the first $n$ positive integers and the Bernoulli numbers
}

\author{
ISTVÁN MOLNÁR
}

Abstract. The first part of this paper presents a method to calculate the sum of the same powers of the first $n$ positive integers which is non-recursive and easy to express algorithmically. The application is demonstrated through several problems, for example by calculating the sum of arithmetic progression of degree $p$. The second part of the paper shows that the discussed procedure can also be used to calculate the Bernoulli numbers, and then, with the help of a known theorem, a link is established between the sum of the same powers of the first $n$ positive integers and the Bernoulli numbers.

Key words and phrases: the sum of the same powers of the first $n$ positive integers, non-recursive method, arithmetic progression, the Bernoulli numbers.

ZDM Subject Classification: D40, F30, F60, H60.

\section{Introduction}

The calculation of the sum of the same powers of the first $n$ positive integers is an interesting and illuminating problem. Below we present a method to do this. The sums in question are calculated with the help of determinants and Cramer's rule, which is a theorem used for linear equations. Even in secondary schools this method - of course first of all in specialized classes and in study groups - may be presented and discussed, depending upon the level of the pupils. Let

$$
S_{p}=1^{p}+2^{p}+3^{p}+\ldots+n^{p}=\sum_{i=1}^{n} i^{p}
$$

Copyright (C) 2011 by University of Debrecen 
where $n$ is a positive integer and $p=0,1,2, \ldots$.

It is easy to calculate $S_{0}$ since obviously $S_{0}=n$.

Our objective is to find a relatively easy-to-use formula for $S_{p}$.

There are different ways to do this, namely:

1) Using Newton's binomial theorem.

Most books and journal articles rely on this method (see, e.g. [1] and [5]). To use this method combinatorial tools are needed (ways to calculate combinations, Newton's binomial theorem).

With this method we describe the $(p+1)$ th power of $(a+1)$ using Newton's binomial theorem. Then, rearranging the result, we get an identity in which we substitute in turns the natural numbers $1,2, \ldots, n$. Adding the terms of the relations that we get this way and reducing the terms of the same nature, we will get a recursive formula. This recursion defines $S_{p}$ as the function of the previous sums $S_{1}, S_{2}, \ldots, S_{p-1}$.

2) Applying other identities.

Just as with the previous method, combinatorial tools are needed here also. To find the recursive pattern we start with unravelling the formula $(x+1) x^{p}-x(x-1)^{p}$ (see, e.g. [3]). By substituting in turns the numbers 1, $2, \ldots, n$ into the identity that we get, and then summing up the results term by term, we will get the recursive formula defining $S_{p}$.

3) Using a matrix.

For this method no combinatorial knowledge or tools are needed. Basic knowledge of matrix arithmetic will help, but there is no essential need for this; instead of the word 'matrix' we can even use the expression 'table'.

We start from the following matrix:

$$
M=\left[\begin{array}{ccccc}
1^{p-1} & 2^{p-1} & 3^{p-1} & \ldots & n^{p-1} \\
1^{p-1} & 2^{p-1} & 3^{p-1} & \ldots & n^{p-1} \\
1^{p-1} & 2^{p-1} & 3^{p-1} & \ldots & n^{p-1} \\
--- & --- & --- & - & --- \\
1^{p-1} & 2^{p-1} & 3^{p-1} & \ldots & n^{p-1}
\end{array}\right]
$$

The essence of this method is that we sum up the elements of the given matrix in different groupings and thus we get the recursion needed. First we sum up the elements of $M$ line by line. Then the summing is done with the help of the upper and lower triangular matrices, and the diagonal matrix assigned to $M$. 
In all these three methods described above we eventually establish a recursive formula. This is precisely the drawback of each of these procedures: because the calculation of a given $S_{p}$ is always done by using the results of $S_{1}, S_{2}, \ldots, S_{p-1}$, and, quite obviously, these results must be calculated beforehand.

However, in the case of the fourth procedure, presented below, there is no need to find previous sums in order to get to the next sum. That is, there is no need to draw up a recursive pattern.

\section{A non-recursive method to calculate $S_{p}$}

Let $k$ be a natural number and $a$ be an arbitrary real number. Start from the identity

$$
\begin{aligned}
(a+1)^{k+1}= & \left(\begin{array}{c}
k+1 \\
0
\end{array}\right) \cdot a^{k+1}+\left(\begin{array}{c}
k+1 \\
1
\end{array}\right) \cdot a^{k}+\left(\begin{array}{c}
k+1 \\
2
\end{array}\right) \cdot a^{k-1}+\ldots+ \\
& +\left(\begin{array}{c}
k+1 \\
k-1
\end{array}\right) \cdot a^{2}+\left(\begin{array}{c}
k+1 \\
k
\end{array}\right) \cdot a+\left(\begin{array}{c}
k+1 \\
k+1
\end{array}\right)
\end{aligned}
$$

from which we get

$$
\begin{aligned}
(a+1)^{k+1}-a^{k+1}= & \left(\begin{array}{c}
k+1 \\
1
\end{array}\right) \cdot a^{k}+\left(\begin{array}{c}
k+1 \\
2
\end{array}\right) \cdot a^{k-1}+\ldots+ \\
& +\left(\begin{array}{c}
k+1 \\
k-1
\end{array}\right) \cdot a^{2}+\left(\begin{array}{c}
k+1 \\
k
\end{array}\right) \cdot a+\left(\begin{array}{c}
k+1 \\
k+1
\end{array}\right)
\end{aligned}
$$

In this equation (1) substitute $a$ in turns with the natural numbers $1,2, \ldots, n$, and sum up the results.

Now

$$
\begin{aligned}
\sum_{i=1}^{n}\left[(i+1)^{k+1}-i^{k+1}\right]= & \sum_{i=1}^{n}\left[\left(\begin{array}{c}
k+1 \\
1
\end{array}\right) \cdot i^{k}+\left(\begin{array}{c}
k+1 \\
2
\end{array}\right) \cdot i^{k-1}+\ldots+\right. \\
& \left.+\left(\begin{array}{c}
k+1 \\
k-1
\end{array}\right) \cdot i^{2}+\left(\begin{array}{c}
k+1 \\
k
\end{array}\right) \cdot i+\left(\begin{array}{c}
k+1 \\
k+1
\end{array}\right)\right]= \\
= & \left(\begin{array}{c}
k+1 \\
1
\end{array}\right) \cdot \sum_{i=1}^{n} i^{k}+\left(\begin{array}{c}
k+1 \\
2
\end{array}\right) \cdot \sum_{i=1}^{n} i^{k-1}+\ldots+ \\
& +\left(\begin{array}{c}
k+1 \\
k-1
\end{array}\right) \cdot \sum_{i=1}^{n} i^{2}+\left(\begin{array}{c}
k+1 \\
k
\end{array}\right) \cdot \sum_{i=1}^{n} i+\left(\begin{array}{c}
k+1 \\
k+1
\end{array}\right) \cdot \sum_{i=1}^{n} 1
\end{aligned}
$$


On the left side we have a telescopic sum which after elimination results in $(n+1)^{k+1}-1^{k+1}$. That is,

$$
\begin{aligned}
(n+1)^{k+1}-1= & \left(\begin{array}{c}
k+1 \\
1
\end{array}\right) \cdot S_{k}+\left(\begin{array}{c}
k+1 \\
2
\end{array}\right) \cdot S_{k-1}+\ldots+ \\
& +\left(\begin{array}{c}
k+1 \\
k-1
\end{array}\right) \cdot S_{2}+\left(\begin{array}{c}
k+1 \\
k
\end{array}\right) \cdot S_{1}+\left(\begin{array}{l}
k+1 \\
k+1
\end{array}\right) \cdot n .
\end{aligned}
$$

Rearrange it to get

$$
\begin{aligned}
(n+1)^{k+1}= & \left(\begin{array}{c}
k+1 \\
k+1
\end{array}\right) \cdot(n+1)+\left(\begin{array}{c}
k+1 \\
k
\end{array}\right) \cdot S_{1}+\left(\begin{array}{l}
k+1 \\
k-1
\end{array}\right) \cdot S_{2}+\ldots+ \\
& +\left(\begin{array}{c}
k+1 \\
2
\end{array}\right) \cdot S_{k-1}+\left(\begin{array}{c}
k+1 \\
1
\end{array}\right) \cdot S_{k} .
\end{aligned}
$$

Let $X_{0}=n+1$.

Assign to relation (2) a linear system of equations that we get by giving $k$ in turns the values $0,1,2, \ldots, p$. Thus we will have this system of equations:

$$
\left\{\begin{aligned}
& n+1=\left(\begin{array}{l}
1 \\
1
\end{array}\right) \cdot X_{0} \\
&(n+1)^{2}=\left(\begin{array}{l}
2 \\
2
\end{array}\right) \cdot X_{0}+\left(\begin{array}{l}
2 \\
1
\end{array}\right) \cdot S_{1} \\
&(n+1)^{3}=\left(\begin{array}{l}
3 \\
3
\end{array}\right) \cdot X_{0}+\left(\begin{array}{l}
3 \\
2
\end{array}\right) \cdot S_{1}+\left(\begin{array}{l}
3 \\
1
\end{array}\right) \cdot S_{2} \\
&------------------- \\
&(n+1)^{p+1}=\left(\begin{array}{c}
p+1 \\
p+1
\end{array}\right) \cdot X_{0}+\left(\begin{array}{c}
p+1 \\
p
\end{array}\right) \cdot S_{1}+\left(\begin{array}{c}
p+1 \\
p-1
\end{array}\right) \cdot S_{2}+\ldots+ \\
&+\left(\begin{array}{c}
p+1 \\
2
\end{array}\right) \cdot S_{p-1}+\left(\begin{array}{c}
p+1 \\
1
\end{array}\right) \cdot S_{p}
\end{aligned}\right.
$$

This is a linear system of equations consisting of $(p+1)$ equations with $(p+1)$ unknowns $\left(X_{0}, S_{1}, S_{2}, \ldots, S_{p}\right) . X_{0}$ is considered unknown only because in this way is it 'simpler' to draw up the equation system, and it is easier to find an algorithm to solve it. 
The matrix derived from the system of equations is a lower triangle matrix, the determinant of which is:

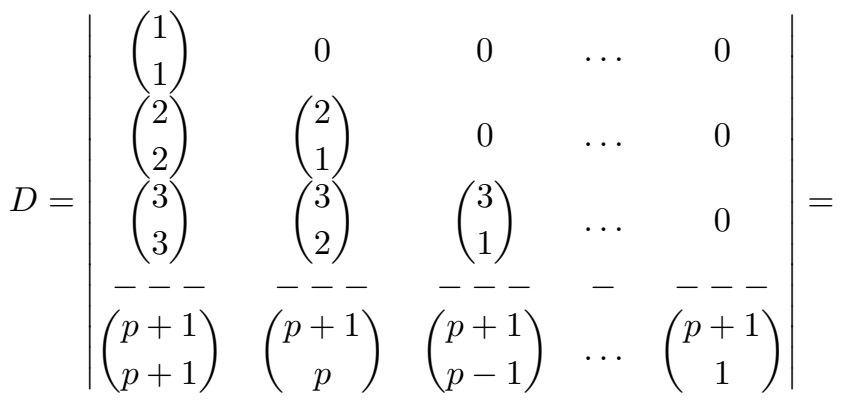

$$
\begin{aligned}
& =\left(\begin{array}{l}
1 \\
1
\end{array}\right) \cdot\left(\begin{array}{l}
2 \\
1
\end{array}\right) \cdot\left(\begin{array}{l}
3 \\
1
\end{array}\right) \cdot \ldots \cdot\left(\begin{array}{c}
p+1 \\
1
\end{array}\right)=(p+1) ! .
\end{aligned}
$$

Since $D=(p+1) ! \neq 0$, Cramer's rule can be applied. We will get the determinants for the corresponding unknowns if in $D$ we substitute in turn the column vectors with the column vectors of the free terms. Thus by calculating the right determinant for each $p$, we will not only get $S_{p}$, but also the $\left(X_{0}, S_{1}, S_{2}, \ldots, S_{p-1}\right)$ sums whose index is lower than $p$.

It is also fairly obvious, however, that if we calculate only $S_{p}$ for each p time after time, then we will get this result even if we do not know the other sums. This shows the greatest advantage of this method compared to other methods, because here to calculate $S_{p}$ we do not have to know the sums $S_{1}, S_{2}, \ldots, S_{p-1}$, i.e., we do not need to draw up a recursive formula.

The determinant of $S_{p}$ is:

$$
D_{p}=\left|\begin{array}{ccccc}
\left(\begin{array}{l}
1 \\
1
\end{array}\right) & 0 & 0 & \ldots & (n+1) \\
\left(\begin{array}{l}
2 \\
2
\end{array}\right) & \left(\begin{array}{l}
2 \\
1
\end{array}\right) & 0 & \ldots & (n+1)^{2} \\
\left(\begin{array}{l}
3 \\
3
\end{array}\right) & \left(\begin{array}{l}
3 \\
2
\end{array}\right) & \left(\begin{array}{l}
3 \\
1
\end{array}\right) & \ldots & (n+1)^{3} \\
--- & --- & --- & - & --- \\
\left(\begin{array}{c}
p+1 \\
p+1
\end{array}\right) & \left(\begin{array}{c}
p+1 \\
p
\end{array}\right) & \left(\begin{array}{c}
p+1 \\
p-1
\end{array}\right) & \ldots & (n+1)^{p+1}
\end{array}\right| .
$$

From Cramer's rule it follows:

$$
S_{p}=\frac{D_{p}}{D}=\frac{1}{(p+1) !} \cdot D_{p},
$$

where $p=1,2,3, \ldots$ (we are not concerned with calculating $X_{0}$ ). 


\section{Applying the relation to some $p$ values}

Here are the results for some actual $p$ values.

If $p=2$, then

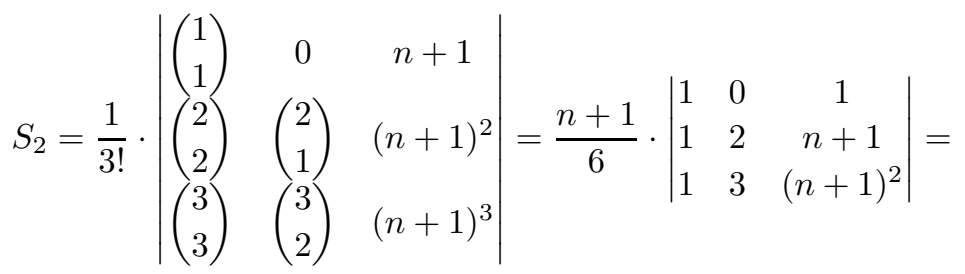

$$
\begin{aligned}
& =\frac{n+1}{6} \cdot\left|\begin{array}{ccc}
1 & 0 & 1 \\
0 & 2 & n \\
0 & 1 & n(n+1)
\end{array}\right|=\frac{n+1}{6} \cdot\left|\begin{array}{cc}
2 & n \\
1 & n(n+1)
\end{array}\right|= \\
& =\frac{n(n+1)}{6} \cdot\left|\begin{array}{cc}
2 & 1 \\
1 & n+1
\end{array}\right|=\frac{n(n+1)}{6} \cdot(2 n+2-1)=\frac{n(n+1)(2 n+1)}{6} .
\end{aligned}
$$

So

If $p=3$, then

$$
S_{2}=1^{2}+2^{2}+3^{2}+\ldots+n^{2}=\frac{n(n+1)(2 n+1)}{6} .
$$

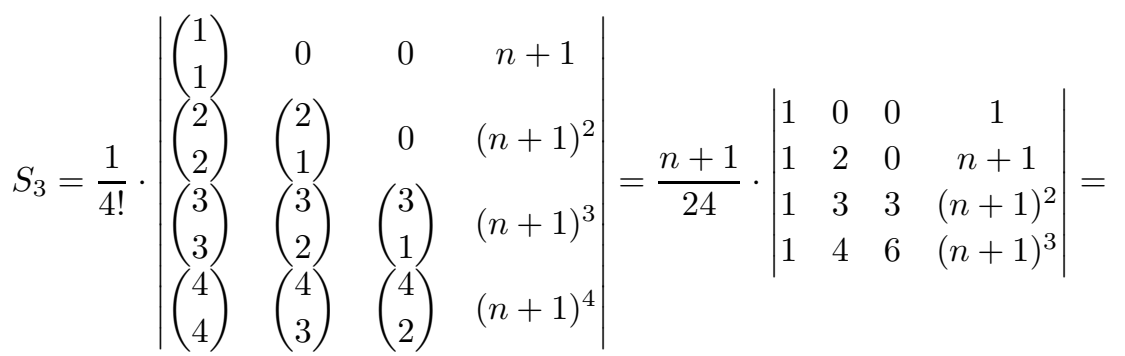

$$
\begin{aligned}
& =\frac{n+1}{24} \cdot\left|\begin{array}{cccc}
1 & 0 & 0 & 1 \\
0 & 2 & 0 & n \\
0 & 1 & 3 & (n+1) \\
0 & 1 & 3 & n(n+1)^{2}
\end{array}\right|=\frac{n+1}{24} \cdot\left|\begin{array}{ccc}
2 & 0 & n \\
1 & 3 & n(n+1) \\
1 & 3 & n(n+1)^{2}
\end{array}\right|= \\
& =\frac{n(n+1)}{8} \cdot\left|\begin{array}{ccc}
2 & 0 & 1 \\
1 & 1 & n+1 \\
1 & 1 & (n+1)^{2}
\end{array}\right|=\frac{n(n+1)}{8} \cdot\left|\begin{array}{ccc}
2 & 0 & 1 \\
1 & 1 & n+1 \\
0 & 0 & n(n+1)
\end{array}\right|=
\end{aligned}
$$




$$
=\frac{n(n+1)}{8} \cdot\left|\begin{array}{cc}
2 & 1 \\
0 & n(n+1)
\end{array}\right|=\frac{n(n+1)}{8} \cdot[2 n(n+1)-0]=\frac{n^{2}(n+1)^{2}}{4} .
$$

So

$$
S_{3}=1^{3}+2^{3}+3^{3}+\ldots+n^{3}=\frac{n^{2}(n+1)^{2}}{4}
$$

These are beyond the syllabuses of ordinary secondary school classes, but - in our experience - they can be taught without reservation in specialized ones (both the mathematics and the method). Depending on the general level of knowledge of the students in the higher years and in study groups, the information about the determinants, Cramer's rule, and the described method can all be presented with ease because these students have by this time acquired the necessary combinatorial skills. Although higher exponents require long calculations along the process, students can perform these without much difficulty after gaining sufficient familiarity with the method.

\section{The generalisation of the method for arithmetic progression}

In the next part we shall see what happens when we work with the consecutive terms of any arithmetic progression.

Take an arithmetic progression with first term $a$ and difference $d(a, d \in \mathbb{R})$. Let us examine what we can say about the sum of the $p$ th powers of the first $n$ terms of the progression, that is, about the sum of the consecutive terms of an arithmetic progression of degree $p$. Let

$$
S_{p}(a, d)=a_{1}^{p}+a_{2}^{p}+a_{3}^{p}+\ldots+a_{n}^{p}=\sum_{i=1}^{n} a_{i}^{p}=\sum_{i=1}^{n}[a+(i-1) \cdot d]^{p},
$$

where $p=0,1,2, \ldots$.

We are again not concerned with calculating $S_{0}(a, d)$, because very obviously $S_{0}(a, d)=n$. Neither does the case need attention when $d=0$, that is when the progression is constant, because then $S_{p}(a, 0)=n \cdot a_{1}^{p}=n \cdot a^{p}$.

So let $d \neq 0$.

The procedure to calculate $S_{p}(a, d)$ is identical with the one that has been introduced above. Start from the identity

$$
\begin{aligned}
a_{i+1}^{k+1}-a_{i}^{k+1}= & \left(\begin{array}{c}
k+1 \\
1
\end{array}\right) \cdot a_{i}^{k} \cdot d+\left(\begin{array}{c}
k+1 \\
2
\end{array}\right) \cdot a_{i}^{k-1} \cdot d^{2}+\ldots+ \\
& +\left(\begin{array}{c}
k+1 \\
k
\end{array}\right) \cdot a_{i}^{1} \cdot d^{k}+\left(\begin{array}{c}
k+1 \\
k+1
\end{array}\right) \cdot d^{k+1},
\end{aligned}
$$


where $i=1,2, \ldots, n$ and $k$ is an arbitrary natural number.

Substituting $i$ in turns with the values $1,2, \ldots, n$, and then summing up the results and making the necessary calculations, we get

$$
\begin{aligned}
(a+n d)^{k+1}-a^{k+1}= & d \cdot\left(\begin{array}{c}
k+1 \\
1
\end{array}\right) \cdot S_{k}(a, d)+\ldots+ \\
& +d^{k} \cdot\left(\begin{array}{c}
k+1 \\
k
\end{array}\right) \cdot S_{1}(a, d)+d^{k+1} \cdot\left(\begin{array}{c}
k+1 \\
k+1
\end{array}\right) \cdot S_{0}(a, d)
\end{aligned}
$$

Rearrange it

$$
\begin{aligned}
(a+n d)^{k+1}-a^{k+1}= & \left(\begin{array}{c}
k+1 \\
k+1
\end{array}\right) \cdot S_{0}(a, d) \cdot d^{k+1}+\left(\begin{array}{c}
k+1 \\
k
\end{array}\right) \cdot S_{1}(a, d) \cdot d^{k}+\ldots+ \\
& +\left(\begin{array}{c}
k+1 \\
1
\end{array}\right) \cdot S_{k}(a, d) \cdot d
\end{aligned}
$$

Assign to relation (6) a linear system of equations generated by giving $k$ in turn the values $0,1,2, \ldots, p$. Thus we will get the following system of equations:

$$
\left\{\begin{array}{l}
(a+n d)^{1}-a^{1}=\left(\begin{array}{l}
1 \\
1
\end{array}\right) \cdot S_{0}(a, d) \cdot d \\
(a+n d)^{2}-a^{2}=\left(\begin{array}{l}
2 \\
2
\end{array}\right) \cdot S_{0}(a, d) \cdot d^{2}+\left(\begin{array}{l}
2 \\
1
\end{array}\right) \cdot S_{1}(a, d) \cdot d \\
(a+n d)^{3}-a^{3}=\left(\begin{array}{l}
3 \\
3
\end{array}\right) \cdot S_{0}(a, d) \cdot d^{3}+\left(\begin{array}{l}
3 \\
2
\end{array}\right) \cdot S_{1}(a, d) \cdot d^{2}+ \\
+\left(\begin{array}{l}
3 \\
1
\end{array}\right) \cdot S_{2}(a, d) \cdot d \\
------------------ \\
(a+n d)^{p+1}-a^{p+1}=\left(\begin{array}{c}
p+1 \\
p+1
\end{array}\right) \cdot S_{0}(a, d) \cdot d^{p+1}+ \\
+\left(\begin{array}{c}
p+1 \\
p
\end{array}\right) \cdot S_{1}(a, d) \cdot d^{p}+\ldots+\left(\begin{array}{c}
p+1 \\
1
\end{array}\right) \cdot S_{p}(a, d) \cdot d
\end{array}\right.
$$

This is a linear system of equations consisting of $(p+1)$ equations with $(p+$ 1) unknowns $\left(S_{0}(a, d), S_{1}(a, d), S_{2}(a, d), \ldots, S_{p}(a, d)\right)$. The determinant of the 


$$
\text { "tmcs-molnar" — 2011/5/14 — 0:02 — page } 99 \text { — \#9 }
$$

matrix belonging to the system of equations is:

$$
\begin{aligned}
D & =\left|\begin{array}{ccccc}
\left(\begin{array}{l}
1 \\
1
\end{array}\right) \cdot d & 0 & 0 & \ldots & 0 \\
\left(\begin{array}{l}
2 \\
2
\end{array}\right) \cdot d^{2} & \left(\begin{array}{l}
2 \\
1
\end{array}\right) \cdot d & 0 & \ldots & 0 \\
\left(\begin{array}{l}
3 \\
3
\end{array}\right) \cdot d^{3} & \left(\begin{array}{l}
3 \\
2
\end{array}\right) \cdot d^{2} & \left(\begin{array}{l}
3 \\
1
\end{array}\right) \cdot d & \ldots & 0 \\
--- & --- & --- & - & --- \\
\left(\begin{array}{l}
p+1 \\
p+1
\end{array}\right) \cdot d^{p+1} & \left(\begin{array}{c}
p+1 \\
p
\end{array}\right) \cdot d^{p} & \left(\begin{array}{c}
p+1 \\
p-1
\end{array}\right) \cdot d^{p-1} & \ldots & \left(\begin{array}{c}
p+1 \\
1
\end{array}\right) \cdot d
\end{array}\right|= \\
& =\left(\begin{array}{l}
1 \\
1
\end{array}\right) \cdot d \cdot\left(\begin{array}{l}
2 \\
1
\end{array}\right) \cdot d \cdot\left(\begin{array}{l}
3 \\
1
\end{array}\right) \cdot d \cdot \ldots \cdot\left(\begin{array}{c}
p+1 \\
1
\end{array}\right) \cdot d=(p+1) ! \cdot d^{p+1} \cdot
\end{aligned}
$$

Since $D=(p+1) ! \cdot d^{p+1} \neq 0$, Cramer's rule can be applied.

The determinant for $S_{p}(a, d)$ is:

$$
D_{p}^{\prime}=\left|\begin{array}{ccccc}
\left(\begin{array}{l}
1 \\
1
\end{array}\right) \cdot d & 0 & 0 & \ldots & (a+n d)^{1}-a^{1} \\
\left(\begin{array}{l}
2 \\
2
\end{array}\right) \cdot d^{2} & \left(\begin{array}{l}
2 \\
1
\end{array}\right) \cdot d & 0 & \ldots & (a+n d)^{2}-a^{2} \\
\left(\begin{array}{l}
3 \\
3
\end{array}\right) \cdot d^{3} & \left(\begin{array}{l}
3 \\
2
\end{array}\right) \cdot d^{2} & \left(\begin{array}{l}
3 \\
1
\end{array}\right) \cdot d & \ldots & (a+n d)^{3}-a^{3} \\
--- & --- & --- & - & --- \\
\left(\begin{array}{c}
p+1 \\
p+1
\end{array}\right) \cdot d^{p+1} & \left(\begin{array}{c}
p+1 \\
p
\end{array}\right) \cdot d^{p} & \left(\begin{array}{c}
p+1 \\
p-1
\end{array}\right) \cdot d^{p-1} & \ldots & (a+n d)^{p+1}-a^{p+1}
\end{array}\right| .
$$

From Cramer's rule it follows:

$$
S_{p}(a, d)=\frac{D_{p}^{\prime}}{D}=\frac{1}{(p+1) ! \cdot d^{p+1}} \cdot D_{p}^{\prime}
$$

where $p=1,2,3, \ldots$.

\section{Example}

If $p=2$, then

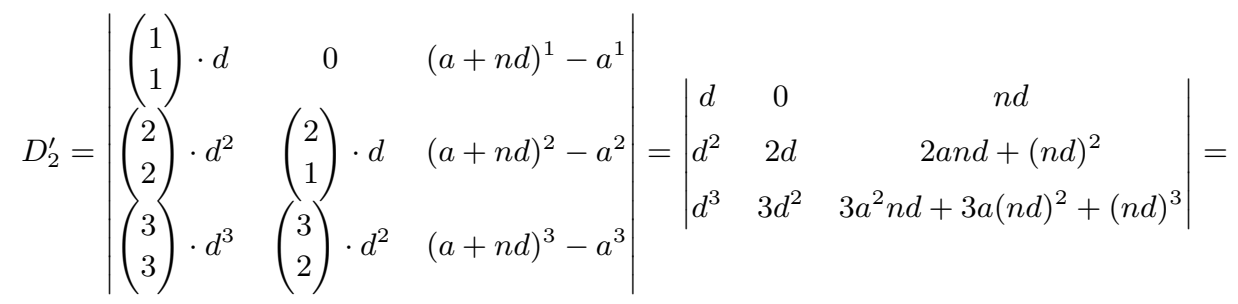




$$
\begin{gathered}
=d \cdot d \cdot n d \cdot\left|\begin{array}{ccc}
1 & 0 & 1 \\
d & 2 & 2 a+n d \\
d^{2} & 3 d & 3 a^{2}+3 a n d+(n d)^{2}
\end{array}\right|=n d^{3} \cdot\left|\begin{array}{ccc}
1 & 0 & 0 \\
d & 2 & 2 a+(n-1) d \\
d^{2} & 3 d & 3 a^{2}+3 a n d+\left(n^{2}-1\right) d^{2}
\end{array}\right|= \\
=n d^{3}\left|\begin{array}{cc}
2 & 2 a+(n-1) d \\
3 d & 3 a^{2}+3 a n d+\left(n^{2}-1\right) d^{2}
\end{array}\right|= \\
=n d^{3}\left[6 a^{2}+6 a n d+2\left(n^{2}-1\right) d^{2}-6 a d-3(n-1) d^{2}\right]= \\
=n d^{3} \cdot\left[2 n^{2} d^{2}+3 n d(2 a-d)+6 a^{2}-6 a d+d^{2}\right] .
\end{gathered}
$$

From which

$$
S_{2}(a, d)=\frac{D_{2}^{\prime}}{D}=\frac{1}{3 ! \cdot d^{3}} \cdot n d^{3} \cdot\left[2 n^{2} d^{2}+3 n d(2 a-d)+6 a^{2}-6 a d+d^{2}\right] .
$$

So

Special cases:

$$
S_{2}(a, d)=\frac{n}{6} \cdot\left[2 n^{2} d^{2}+3 n d(2 a-d)+6 a^{2}-6 a d+d^{2}\right] .
$$

$a=1, d=1$

$$
\begin{aligned}
& \quad S_{2}(1,1)=1^{2}+2^{2}+3^{2}+\ldots+n^{2}=\frac{n}{6} \cdot\left(2 n^{2}+3 n+1\right)=\frac{n(n+1)(2 n+1)}{6} . \\
& a=1, d=2 \\
& \quad S_{2}(1,2)=1^{2}+3^{2}+5^{2}+\ldots+(2 n-1)^{2}=\frac{n}{6} \cdot\left(8 n^{2}-2\right)=\frac{n\left(4 n^{2}-1\right)}{3} .
\end{aligned}
$$

$a=1, d=3$

$S_{2}(1,3)=1^{2}+4^{2}+7^{2}+\ldots+(3 n-2)^{2}=\frac{n}{6} \cdot\left(18 n^{2}-9 n-3\right)=\frac{n}{2} \cdot\left(6 n^{2}-3 n-1\right)$.

$a=3, d=4$

$S_{2}(3,4)=3^{2}+7^{2}+11^{2}+\ldots+(4 n-1)^{2}=\frac{n}{6} \cdot\left(32 n^{2}+24 n-2\right)=\frac{n}{3} \cdot\left(16 n^{2}+12 n-1\right)$.

Generalization for arithmetical progression can be one of the topics taught here, though the calculations will stretch even longer (however they are quite rewarding). The examination of some special cases, on the other hand, can highlight a number of interesting relations.

\section{The Bernoulli numbers}

Following the same train of thought the Bernoulli numbers can also be calculated. In one interpretation the Bernoulli numbers are the coefficients of the 
Taylor expansion of the function $\frac{x}{e^{x}-1}$ :

$$
\frac{x}{e^{x}-1}=\sum_{k=0}^{\infty} \frac{B_{k}}{k !} \cdot x^{k}
$$

where $B_{k}$ 's are the Bernoulli numbers (for more details see, e.g. [4]).

According to another interpretation (see, e.g. [7]), we will get the first $(p+1)$ Bernoulli numbers $\left(B_{0}, B_{1}, B_{2}, \ldots, B_{p}\right)$, if we solve the following system of equations:

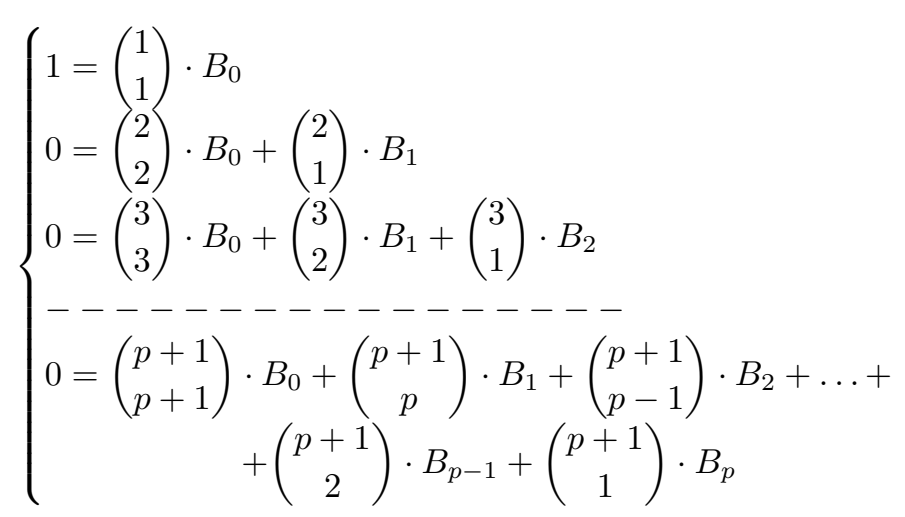

The determinant of the matrix belonging to the system of equations (8) is:

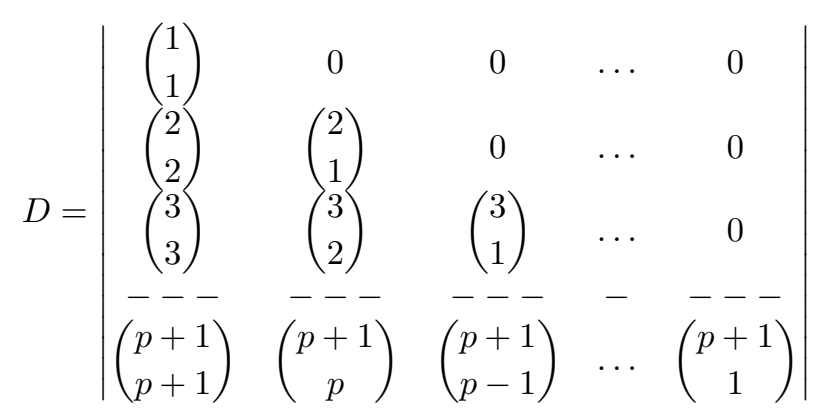

$$
\begin{aligned}
& =\left(\begin{array}{l}
1 \\
1
\end{array}\right) \cdot\left(\begin{array}{l}
2 \\
1
\end{array}\right) \cdot\left(\begin{array}{l}
3 \\
1
\end{array}\right) \cdot \ldots \cdot\left(\begin{array}{c}
p+1 \\
1
\end{array}\right)=(p+1) !
\end{aligned}
$$

Since $D=(p+1) ! \neq 0$, Cramer's rule can be applied.
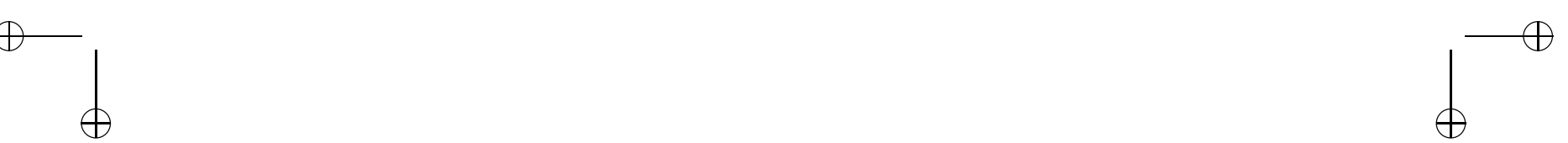
The determinant for $B_{p}$ is:

$$
D_{p}^{\prime \prime}=\left|\begin{array}{ccccc}
\left(\begin{array}{l}
1 \\
1
\end{array}\right) & 0 & 0 & \ldots & 1 \\
\left(\begin{array}{l}
2 \\
2
\end{array}\right) & \left(\begin{array}{l}
2 \\
1
\end{array}\right) & 0 & \ldots & 0 \\
\left(\begin{array}{l}
3 \\
3
\end{array}\right) & \left(\begin{array}{l}
3 \\
2
\end{array}\right) & \left(\begin{array}{l}
3 \\
1
\end{array}\right) & \ldots & 0 \\
--- & --- & --- & - & --- \\
\left(\begin{array}{c}
p+1 \\
p+1
\end{array}\right) & \left(\begin{array}{c}
p+1 \\
p
\end{array}\right) & \left(\begin{array}{c}
p+1 \\
p-1
\end{array}\right) & \ldots & 0
\end{array}\right|
$$

So from Cramer's rule it follows:

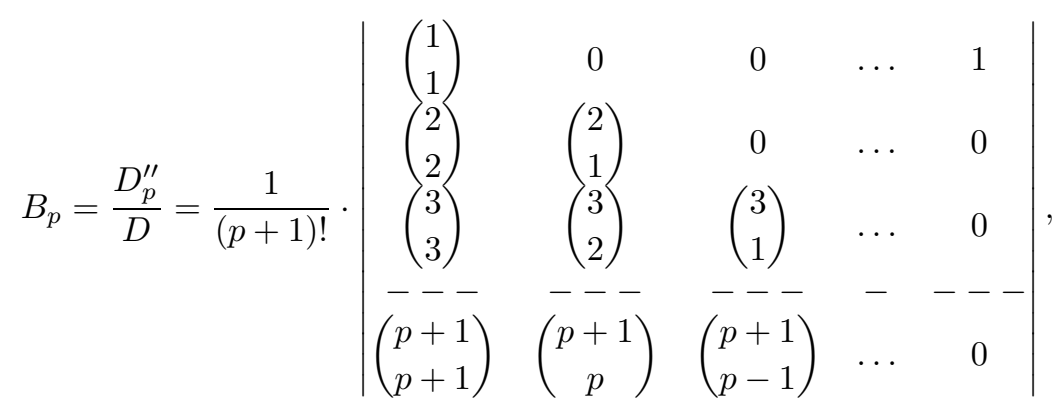

where $p=1,2,3, \ldots$ (we are not concerned with calculating $B_{0}$, because it immediately follows from the first equation that $B_{0}=1$ ).

\section{Example}

Calculate $B_{4}$.

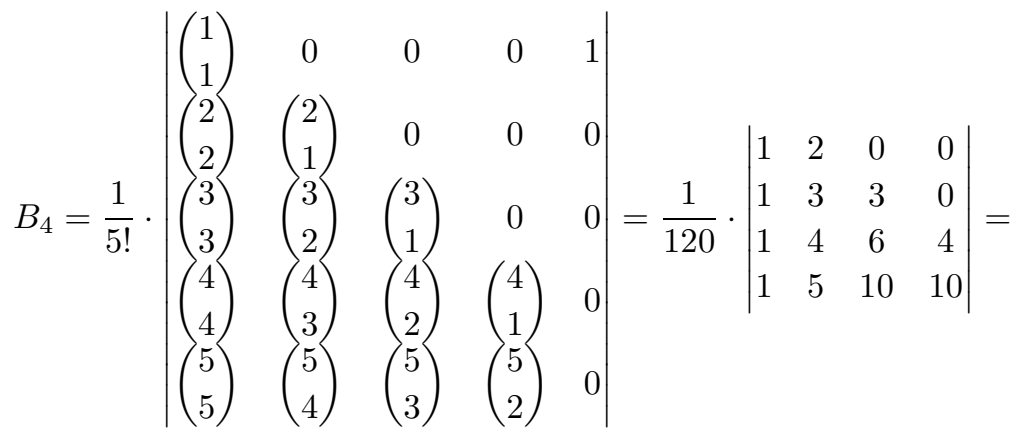




$$
\begin{aligned}
& =\frac{1}{120} \cdot\left|\begin{array}{cccc}
1 & 2 & 0 & 0 \\
0 & 1 & 3 & 0 \\
0 & 2 & 6 & 4 \\
0 & 3 & 10 & 10
\end{array}\right|=\frac{1}{120} \cdot\left|\begin{array}{ccc}
1 & 3 & 0 \\
2 & 6 & 4 \\
3 & 10 & 10
\end{array}\right|=\frac{1}{120} \cdot\left|\begin{array}{ccc}
1 & 0 & 0 \\
2 & 0 & 4 \\
3 & 1 & 10
\end{array}\right|= \\
& =\frac{1}{120} \cdot\left|\begin{array}{cc}
0 & 4 \\
1 & 10
\end{array}\right|=\frac{1}{120} \cdot(0-4)=-\frac{1}{30} .
\end{aligned}
$$

The values of the first few Bernoulli numbers:

$$
B_{0}=1 ; B_{1}=-\frac{1}{2} ; B_{2}=\frac{1}{6} ; B_{3}=0 ; B_{4}=-\frac{1}{30} ; B_{5}=0 ; B_{6}=\frac{1}{42} .
$$

The next theorem links up the sum of the $p$ th power of the first $n$ positive integers with the Bernoulli numbers.

\section{Theorem (Jakob Bernoulli)}

The sum of the $p$ th $\left(p \in \mathbb{Z}^{+}\right)$power of the first $n$ positive integers can be generated this way:

$$
S_{p}=\sum_{i=1}^{n} i^{p}=\frac{1}{p+1} \cdot \sum_{k=0}^{p}\left(\begin{array}{c}
p+1 \\
k
\end{array}\right) \cdot B_{k} \cdot(n+1)^{p+1-k} .
$$

The proof of the theorem, which is based on the method of mathematical induction, can be found, for example, in [2].

Example

If $p=2$, then

$$
\begin{aligned}
S_{2} & =\frac{1}{3} \cdot \sum_{k=0}^{2}\left(\begin{array}{l}
3 \\
k
\end{array}\right) \cdot B_{k} \cdot(n+1)^{3-k}= \\
& =\frac{1}{3} \cdot\left[\left(\begin{array}{l}
3 \\
0
\end{array}\right) \cdot 1 \cdot(n+1)^{3}+\left(\begin{array}{l}
3 \\
1
\end{array}\right) \cdot\left(-\frac{1}{2}\right) \cdot(n+1)^{2}+\left(\begin{array}{l}
3 \\
2
\end{array}\right) \cdot\left(\frac{1}{6}\right) \cdot(n+1)\right]= \\
& =\frac{n+1}{3} \cdot\left[(n+1)^{2}-\frac{3}{2} \cdot(n+1)+\frac{1}{2}\right]= \\
& =\frac{n+1}{6} \cdot\left(2 n^{2}+4 n+2-3 n-3+1\right)=\frac{n+1}{6} \cdot\left(2 n^{2}+n\right)= \\
& =\frac{n(n+1)(2 n+1)}{6} .
\end{aligned}
$$


If $p=3$, then

$$
\begin{aligned}
S_{3} & =\frac{1}{4} \cdot \sum_{k=0}^{3}\left(\begin{array}{l}
4 \\
k
\end{array}\right) \cdot B_{k} \cdot(n+1)^{4-k}= \\
& \frac{1}{4} \cdot\left[\left(\begin{array}{l}
4 \\
0
\end{array}\right) \cdot 1 \cdot(n+1)^{4}+\left(\begin{array}{l}
4 \\
1
\end{array}\right) \cdot\left(-\frac{1}{2}\right) \cdot(n+1)^{3}+\left(\begin{array}{l}
4 \\
2
\end{array}\right) \cdot\left(\frac{1}{6}\right) \cdot(n+1)^{2}+0\right]= \\
& =\frac{(n+1)^{2}}{4} \cdot\left[(n+1)^{2}-2(n+1)+1\right]=\frac{(n+1)^{2}}{4} \cdot(n+1-1)^{2}= \\
& =\frac{n^{2}(n+1)^{2}}{4} .
\end{aligned}
$$

We want to note that in [6] there are nearly forty explicit formulas for calculating Bernoulli numbers. With the help of these, from the above theorem, many nonrecursive formulas can be made to calculate the sum of the same powers of the first $n$ positive integers.

\section{Summary}

In this paper we approached the problem of calculating the sum of the same powers with linear algebraic means. For this we first drew up a 'suitable' relation and then assigned to it a linear system of equations. Solving the system of equations with Cramer's rule, we obtained a closed formula for the sum $S_{p}$, which is derived from the calculation of a determinant. The method could be generalized for arithmetical progression as well. The use of a linear system of equations in solving the problem also creates a link with the Bernoulli numbers. We have shown that the method we presented can be applied to calculate Bernoulli numbers. We established a connection between the sum of the $p$ th powers of the first $n$ positive integers and the Bernoulli numbers. The method presented here and its applications - as can be seen - help to illustrate how 'bridges' may be built between the different areas of mathematics, and pointing this out may also be an important goal when teaching mathematics at a higher level, both in secondary and in higher education.

\section{References}

[1] M. Bencze, About the sum $[\sqrt[k]{1}]^{p}+[\sqrt[k]{2}]^{p}+\cdots+[\sqrt[k]{n}]^{p}$, Octogon Mathematical Magazine, Braşov 9, no. 1A (April 2001), 126-135. 
[2] http://fermatslasttheorem.blogspot.com/2006/10/bernoulli-numbers.html.

[3] S. Kiss, Kísérlet az $1^{k}+2^{k}+3^{k}+\ldots+n^{k}$ összeg zárt alakban való előállítására, Matlap, Kolozsvár 9 (2008), 327-330.

[4] E. D. Knuth, A számítógép-programozás müvészete 1, Müszaki Könyvkiadó, Budapest, 1994, 128-133.

[5] Gy. Pólya, A problémamegoldás iskolája I., Tankönyvkiadó, Budapest, 1967, 79-80.

[6] L. Saalschütz, Vorlesungen über die Bernoullischen Zahlen; ihren Zusammenhang mit den Secanten-Coefficienten und ihre wichtigeren Anwendungen, Julius Springer, Berlin, 1893.

[7] P. Szász, A differenciál- és integrálszámítás elemei 1., Typotex Kiadó, Budapest, 2000, 680-685.

ISTVÁN MOLNÁR

FACULTY OF ECONOMICS

SZENT ISTVÁN UNIVERSITY

BAJZA UTCA 33.

H-5600 BÉKÉSCSABA

HUNGARY

E-mail: molnar.istvan@gk.szie.hu

(Received October, 2010) 